\title{
Adaptive CAC with Traffic Flows Classification for IEEE 802.16 Networks
}

\author{
Marwen Abdennebi, Yacine Ghamri-Doudane and Yan Li \\ École Nationale Supérieure d'Informatique pour l'Industrie et l'Entreprise (ENSIIE) \\ 1 Sq. de la résistance, 91025 Evry Cedex - France \\ \{abdennebi,ghamri,li\}@ensiie.fr
}

\begin{abstract}
In this paper, we propose an adaptive Call Admission Control for IEEE 802.16 scheduled flows. As the WiMAX defined services aim to adapt the bandwidth grant to the data rate of accepted sessions, a powerful CAC that takes into account flows characteristics and their heterogeneities must be used. Our proposal aims to take advantage of the variability of traffics and to adapt the CAC according to the characteristics of incoming flows. We apply this adaptive CAC to the case of video traffics using measurements done by the BS to guess and classify flows according to the similarities in their behaviours and then adapt the system model parameters that will be used by the CAC. In this purpose we use a Markovian model adapted for each flow instead of a generic one. Performance evaluation is given to demonstrate the interest of our proposal.
\end{abstract}

\section{INTRODUCTION}

The Call Admission Control (CAC) is a very important function for WiMAX IEEE 802.16 [1] system in order to ensure that once a session is accepted by the system, the QoS will be guaranteed for the whole session duration. For the Unsolicited Grant Service (UGS) the CAC could be seen as an algorithm that compares the fixed bandwidth requested with the availability in the MAC frame and simply grant it if this availability is confirmed. However, for the particular case of real-time Polling Service (rtPS), this scheme must take into account the mean estimation and the probabilities of each bandwidth request and its variability in time.This estimation should be based on analytical sources traffic models. System modelling will give the probabilities of each traffic state and hence will allow the operator to guess the probabilities of each bandwidth request related to bitrate variations.

Usually, the corresponding algorithms use fixed system models, with one model per traffic source. However, for each traffic source, different source model could be considered by using different sets of parameters, but the values of those parameters should be appropriate for the considered system. Moreover, we face another problem when we are dealing with live shows and IPTV flows for which we have no precise information about the statistical behaviour of the bandwidth needed. In this case, a typical video model with fixed parameters will not be accurate for the flow characteristics. Many proposals were done in this area [2][3] but the measurements that they propose to integrate are used to adapt the parameters of the traffic descriptors but not for the flow classification.

Our proposal consists in using, in addition to the parameters adaptation, combined traffic modelling and flows measurements for the traffic sources classification according to their similar statistical behaviours. This will lead to also adapt the CAC. This adaptation should be realised according to the incoming traffic thanks to measurements performed on them. The BS should be in charge of those measurements, in both uplink and downlink.

In our case, we will illustrate this proposal using the example of video traffics. This case is especially interesting as the traffic flow rate and its variations are related to the motion level of the videos. We will then demonstrate that the combined models will be more accurate than a single one.

Hence, in the following, we will first introduce the background study and the existing IEEE 802.16 services and CAC. Then, section III describes our proposed adaptive CAC system. In section IV, we will describe the adaptation proposed, the system model used and how it should use the measurements done at the BS level as well as the impact on the CAC. We will give some examples in the performance evaluation section (section V) to show the benefits of our proposal. Finally, in section VI, we end with a conclusion and the future research directions to improve and develop this work.

\section{BACKGROUND}

The Call Admission Control (CAC) is an important function in WiMAX networks as it must be defined for each service and application. While for UGS, it simply consists in admitting only sessions that fit the mean available bandwidth, for rtPS (and even for extend rtPS, ertPS), the bitrate requested and its variation should be taken into account before admitting a session.

In fact, in rtPS, the regular polling of admitted sources implies various bandwidth requests in time and space related to the throughput variations of the corresponding streams. It means that almost all requests must be granted in order to satisfy the QoS in term of drop rates and/or delays. Actually, for each time interval, the bandwidth requested should be compared to the available one (in term of time slots and/or symbols in frame) to ensure the grant.

Hence, the CAC function has to be aware of the traffic models of requesting sources as those models will give the 
bitrate variations and hence the bandwidth requests for the WiMAX frames. As the traffic models used will finally give the distribution of the bandwidth requests (in term of probabilities), it is very important that those models should be as accurate as possible, for both single and aggregated traffic sources. That consists in first computing, for each time interval, the probability of the bandwidth request related to each flow, this using the simple traffic model for the corresponding source. Then, it should be compared with the available bandwidth for this service in the WiMAX frames that will be given using the aggregated traffic model for all admitted sources in rtPS. If the grant is possible for the defined number of frames (given from the polling interval), the bandwidth will be allocated, else, the session must delay its transmission or even drop packets.

Usual CAC algorithms often use the same model for a single video stream. The most commonly used CAC will compute the probabilities states of the modelled system and then determine the maximum number of sources that should be accepted [5] [6]. The main goal is then to ensure that the QoS condition is respected for the traffic sources in term of bandwidth/rate, delay, jitter and drops. The system can also accept or reject sessions "on the fly", according to bandwidth availability, but, this time, the system will not be able to accurately predict variations for this bandwidth and hence the guarantee would not be respected.

\section{SYSTEM DESCRIPTION}

\section{A. Adaptive CAC process}

Our goal is to adapt the dimensioning and/or CAC according to the characteristics of the incoming flows by taking into account their different motion levels. We will then split the incoming traffic into different classes according to their rate levels statistics. Hence, the CAC will be done according to the repartition of each class and its importance (number of flows belonging to this class). In this purpose, measurements of statistical behaviour of video traffics should be realised by the BS to obtain classification. Note that this proposal can be added to the measurements based $\mathrm{CAC}$ which adapt the flow characteristics to the measurements. Actually, in our case, the measurements will be mainly done to classify flows to obtain a refined aggregated model instead of using only a simple model (assuming that this one could also be adapted to the measurements).

For video traffic, rates and rate variations differ from one flow to another according to the motion included in each video. The model to be used should then be related to the level of the motions itself. We choose 3 levels of motion as recommended in [7] [8] which means 3 simple models M1, M2 and M3 for each flow category that will lead to a 3D aggregated video model. The dimensions N1, N2, N3 of this 3D model will be of course related to the number of flows belonging to each video motion level category M1, M2, M3.

Even if our CAC will behave similarly in a very big time scale as a non adaptive case, our proposed system will result in variability in the flow admissions in time and space. In our case, each flow is first treated apart and the measurements will allow classifying it in the appropriate category.

For the considered case of video flows, those measurements should be done in the scene time scale, which means at least seconds/minutes. We first quantize the flow to map with discrete rate level models, then find the probability density function (rate levels for example) of the flow and finally classify it. After the measurements and classification, we are able to update the flow repartition rates, then the global system model parameters and hence update the CAC function.

We can describe the process and its steps by the figure 1 below.

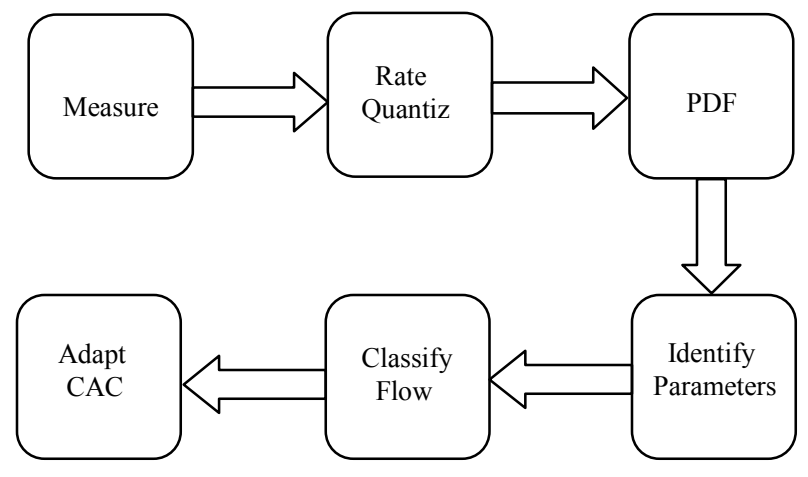

Figure 1. Adaptive CAC process description

As stated earlier, we will propose to apply our scheme to the particular case of video traffic which has important rate level variability according to the motion level. The quantization is especially important if we consider the use of discrete rate level models. In this case the quantization level should map an analytical model dimension used for the rate level description.

The final adaptive CAC will consist in the determination for each update interval, the maximum number of sources that could be admitted in the system assuming the $802.16 \mathrm{rtPS}$ parameters like the available bandwidth for the service, the modulation used depending on the SNR of each mobile $i$ (from $\mathrm{N})$, the scheduling type and the grant interval in term of number MAC frames per request $p$ and frame duration $f_{d}$.

This will result, in given the CAC, for the entire rtPS service and for each time interval, the bandwidth requests $\sum_{i=1}^{N} \frac{R a t e(t)}{p \cdot f d}$ and the available bandwidth $\sum_{i=1}^{N}$ rate $[\bmod (S N R(i))]$ (assuming one session for each mobile, i.e., $N$ sessions for $N$ mobile stations).

\section{B. Individual Source Traffic Model}

The individual source model we propose to use in order to illustrate the proposal is a single dimension Markovian rate based chain. This model maps fairly good with time scale aggregated frames where the request concern more than one WiMAX frames like the one we consider in rtPS service. The quantization should then be in the same time scale.

For video flows, we propose to use for the validation testing the model as described in the figure 2 below. However, it is important to note that other models could be used and mapped accurately using the same measurements but with other appropriate quantization and statistical analysis.

This type of modelling studies the statistical properties of the 
video rate $D(t)$. A video flow can be characterized by the mean rate $E[D]$, the variance of the rate $\operatorname{Var}[D]$, and the correlation function $\operatorname{corr}(\tau)=e^{-a \tau}$. This behaviour can be approximated by the MMPP-M+1 process as shown in Figure 2.
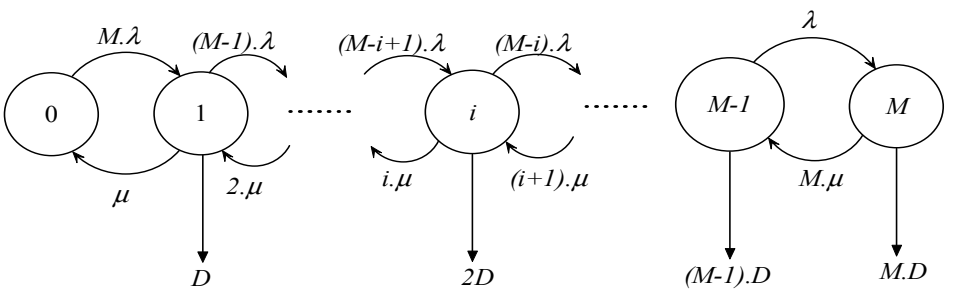

Figure 2. Markov description of single video rate process

The process parameters $\lambda$ and $\mu$ are respectively the mean arrival and service rates from one quantized bitrate to another where $\mathrm{D}$ is the transition between the states and M.D the maximum bitrate.

Each state above corresponds to a quantized value of the bitrate. The MMPP calculations give:

$$
\begin{aligned}
& E[D]=M D \frac{\lambda}{\lambda+\mu} \\
& \operatorname{Var}(D)=M \cdot D^{2} \frac{\lambda \mu}{(\lambda+\mu)^{2}} \\
& \operatorname{Corr}(\tau)=e^{-(\lambda+\mu) \tau}
\end{aligned}
$$

The state probabilities are obtained by solving the following linear system of equations:

$$
\begin{aligned}
\pi_{i}=\left(\begin{array}{c}
N \\
i
\end{array}\right) \cdot \frac{\rho^{i}}{(1+\rho)^{N}} \\
\text { with } \rho=\frac{\lambda}{\mu} \text { and } \quad \pi_{0}=\frac{1}{(1+\rho)^{N}}
\end{aligned}
$$

This discrete Markov model is a large scale model (GoP scale minimum) with lower variations. The statistical properties of the video rate $D(t)$ is described with discrete values following the steps $(\operatorname{Max}(\mathrm{D}) / \mathrm{M})$ in $\mathrm{M}+1$ quantized levels.

Once the flow quantized and the PDF function determined, we can map it to the performed measurements.

\section{MEASUREMENTS FOR SYSTEM MODELLING}

\section{A. Measurements for Sources Classification}

For each accepted flow, the measurements are done in order to guess its statistical characteristics. The goal of this step is to classify the video session into the appropriate chain model from the 3 defined categories by mapping it with the parameters one of the Markov chains corresponding to the category proposed.

The measurement process should update the system model parameters, and hence the CAC in term of possible admissions. The following figure shows an example of a video flow measured at the BS level and the obtained variation of M.D parameters. We take the example of a soccer game video provided in [10]. We can see that the parameters detection convergence begins at the $5000^{\text {th }}$ frame, which corresponds to about 3 minutes of measurements.

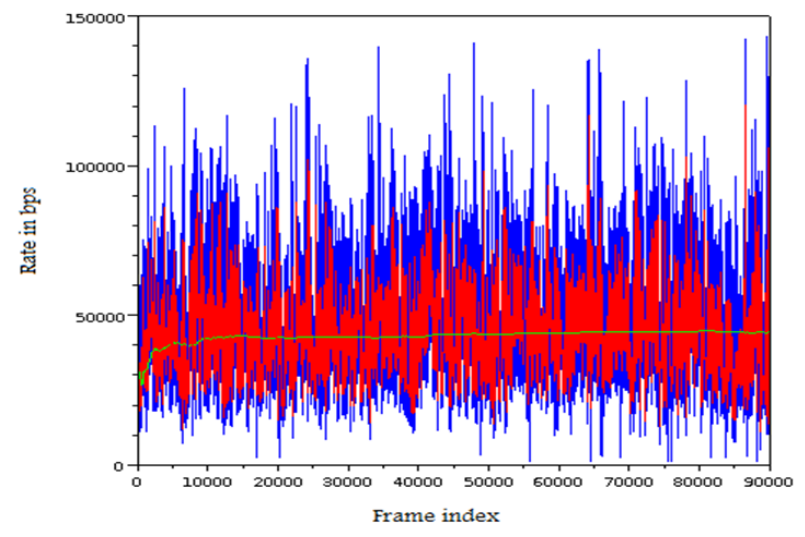

Figure 3. Example of request bandwidth (blue), quantization (red) and convergence of the mean value (green) $V s$ the frame number

We apply large scale, rate based models to model the system. The final model is intended to give the states probabilities of the aggregated video traffic models (one model per traffic category).

It is important to note that for each flow measurements should be performed repeatedly to update the system. This is done in the time scale of the scene mean duration for $\mathrm{CAC} /$ Dimensioning time update, not for $\mathrm{CAC}$ function.

Actually what we mean by convergence is the fact that the measured parameters reach a fixed value and does not diverge for more that $n$ frames. For the average value for example, it means that we can update the $\mathrm{CAC}$ after the measurement of one flow, until reaching this value.

$$
\left|\sum_{\text {frame } k=1}^{i} \frac{1}{k} \operatorname{rate}(k)-\sum_{\text {frame } j=1}^{i-1} \frac{1}{j} \operatorname{rate}(j)\right|<l
$$

where lis the converge limit.

In the case of rtPS scheduling, the number of frames related to the request should be also taken into account as this number is used as a minimum time granularity for the quantization step. It means that this value is directly related to the number of bytes that the request concerns, and hence also the states probabilities of each considered flow.

Once the convergence of the measurements achieved, we obtain the probabilities of each rate state through a histogram of the rate levels. Those values will allow us to classify the flow in the appropriate category, and then update the system model parameters. In addition to the PDF function, note that we also need the state duration values, in order to guess the value of $\lambda$ and $\mu$. Once obtained, we classify the flow in the appropriate traffic model according to the closest measured values of $\lambda$ and $\mu$.

Equations (6) and (7) are used to guess the individual flow characteristics, using the Markov behaviour of the quantized rate levels.

The mapping is then deduced after the measurement of mean durations in each state $i$ which is exponentially distributed with a mean duration $T i$ :

$$
T_{i}=\frac{1}{(n-i) \lambda+i . \mu}
$$

and of course the occurrence of each quantized data rate: 


$$
\pi_{i}=\left(\begin{array}{c}
N \\
i
\end{array}\right) \cdot \frac{\rho^{i}}{(1+\rho)^{N}}
$$

As we see, it will then consist in a simple linear system of 2 equations with $\lambda$ and $\mu$ as the variables to determine.

\section{B. System Modelling and CAC Adaptation}

Once the classification obtained and the Markov chain solved, we will have the 3 bounds of the 3 dimensions (3D) Markov chain. For each time interval $t$ (usually scene level), after the system is updated with flows measurements and dimensions of the chain which depends on the repartition of the flows categories, we obtain the updated states probabilities $\pi_{i, j, k}$ values for the 3D model.

This 3D markov model will be solved using the N1, N2 and $\mathrm{N} 3$ bounds corresponding respectively to the number of flows that could be accepted in each traffic category M1, M2 and M3 with the measured parameters values $\lambda_{1,2,3}$ and $\mu_{1,2,3,}$. Figure 4 gives the aggregated chain that describes this system, with $i, j$ and $k$ the numbers of flow matching to rates models M1, M2 and M3 respectively.

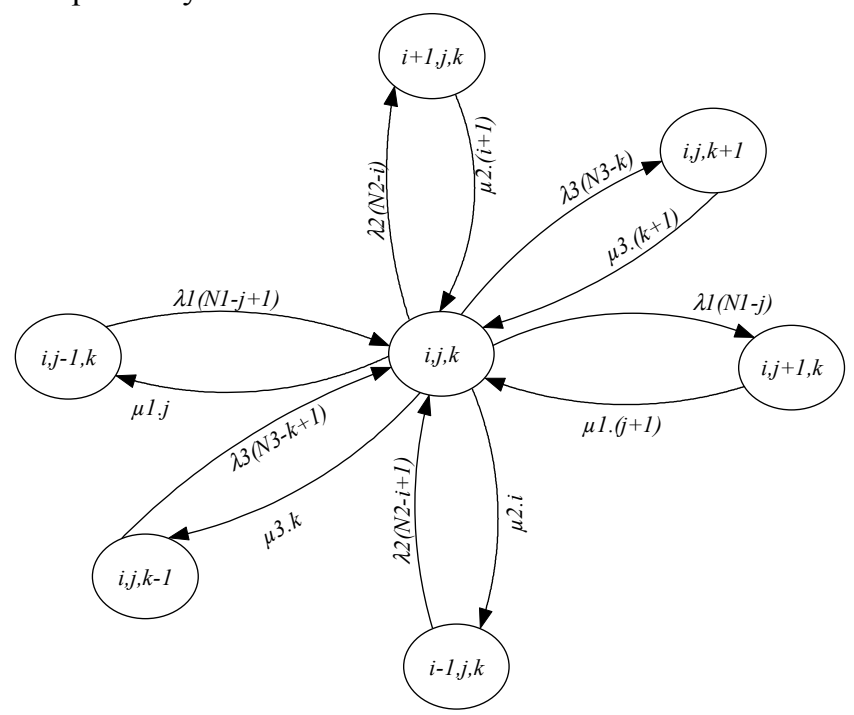

Figure 4. 3D Markov system model with $i, j, k$ the number of flows in each model category M1, M2, M3

This Markov chain should be solved with the appropriate bounds and thanks to steady state equations (8) and (9) below.

$$
\begin{aligned}
& \pi_{i, j, k} \cdot\left(j \cdot \mu_{1}+(N 1-j) \cdot \lambda_{1}+i \cdot \mu_{2}+(N 2-i) \cdot \lambda_{2}+k \cdot \mu_{3}+(N 3-k) \cdot \lambda_{3}\right)= \\
& \quad \pi_{i, j-1, k} \cdot(N 1-j+1) \cdot \lambda_{1}+\pi_{i-1, j, k}(N 2-i+1) \cdot \lambda_{2}+\pi_{i, j, k-1} \cdot(N 3-k+1) \cdot \lambda_{3} \\
& \quad+\pi_{i, j+1, k} \cdot(j+1) \cdot \mu_{1}+\pi_{i+1, j, k} \cdot(i+1) \cdot \mu_{2}+\pi_{i, j, k+1} \cdot(k+1) \cdot \mu_{3}
\end{aligned}
$$

We should of course add the conservation equation:

$$
\sum_{i=1}^{N 1} \sum_{j=1}^{N 2} \sum_{k=1}^{N 3} \pi_{i, j, k}=1
$$

We choose to numerically solve this system (in our case using the Matlab solver for equations (8) and (9)). The value $\mathrm{N}$ of the maximum number of admitted sources will be determined through the N1, N2 and N3 parameters which are the bounds of the 3D Markov chain.

Actually, we should find $\mathrm{N}$ that satisfy, in addition to the conservation equation $N=N 1+N 2+N 3$, the following equation system.

$$
\left\{\begin{array}{l}
N 1=N \cdot \eta_{1} \Rightarrow N=\frac{N 1}{\eta_{1}} \\
N=\frac{N 2}{\eta_{2}} \\
N=\frac{N 3}{\eta_{3}}
\end{array}\right.
$$

Where $\eta_{1}, \eta_{2}, \eta_{3}$ represent the relative number of sources of each category of classified video traffic respectively.

And of course, the final equation will be given by the CAC decision that should satisfy the QoS threshold for:

The mean bandwidth:

$$
B W=\sum_{i=1}^{N 1} \sum_{j=1}^{N 2} \sum_{k=1}^{N 3} \pi_{i, j, k} \cdot[\operatorname{rate}(i)+\operatorname{rate}(j)+\operatorname{rate}(k)]
$$

And the limitation given by the maximum bandwidth $B W_{\max }$ (in term of the maximum of equivalent admitted sources):

$\operatorname{prob}\left(B W_{\max }\right)=\sum_{i=1}^{N 1} \sum_{j=1}^{N 2} \sum_{k=B W_{\max }-(i+j)}^{N 3} \pi_{i, j, k} \cdot \operatorname{rate}(i, j, k)<$ Thres

That should be limited by the threshold value Thres. In fact, this probability is directly related and should be limited by this QoS threshold which depends on users and operators. For the case where the CAC is not adaptive, we will also have the same kind of limitation, with no distinction of categories $i, j$ and $k$, this to allow us to compare both cases in the performance evaluation section.

Hence this probability limitation could be set according to objective parameters like delay, jitter and drop rates and/or to more video-specific like PSNR. We will then be able to set the value of the threshold according to the performance testing.

\section{PERformance EVAluation}

In this part, we propose to validate the proposed adaptive CAC and we evaluate the performances. The validation testing results should illustrate that the CAC can accept more sessions with the same bandwidth allocated and with no QoS degradation. In the other hand, the performance testing should demonstrate that the mean QoS is enhanced for each accepted flow if the adaptive CAC accepts the same number of sessions.

Once the parameters of the system model known, we conduct a set of simulations using NS-2 tool to demonstrate whether our proposed CAC confirms our expectations or not. In this purpose, we use video flows downloaded from [9] [10]. Each video flow is described in a file containing the data frame type (I, B or P), the frame size and time the frame is generated. Those frames are first analysed under Matlab. Then, the parameters are obtained and the 3D Markov chain is determined through solving the system equations. We then find the probabilities of the outbound states which should be set as under the threshold. 
Then, we validate using NS2 by calculating the mean throughput of those video flows and check if the CAC set gives enough bandwidth.

\section{A. Flow Traces Analysis and Classification}

As described above, the first step is to make measurements on the incoming video flows. We used the MPEG4 video traffic flows provided by [10]. Those video are related to various traffic type, from very high data rates like those obtained with soccer matches to medium and low rate levels like those obtained with talks.

Measurements are done to compute the PDF function with the corresponding level durations, then determining the $\lambda$ and $\mu$ values. Once the parameters given, we find the $\eta_{1}, \eta_{2}, \eta_{3}$ values and finally update the Markov chain parameters.

An example of a high speed soccer game video flow is given in the figure 5 .

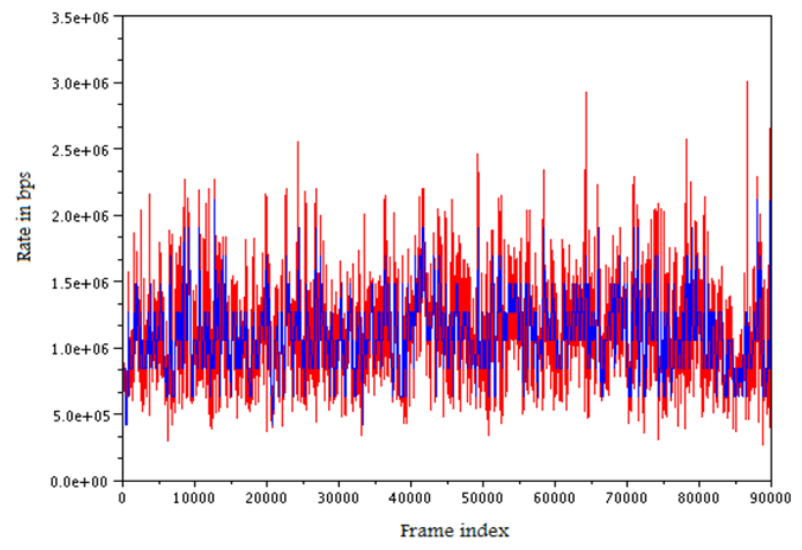

Figure 5. Rate (red) and quantized rate (blue)

Once the convergence of parameters measurements obtained, we obtain the corresponding PDF function, as in Figure 6 below.

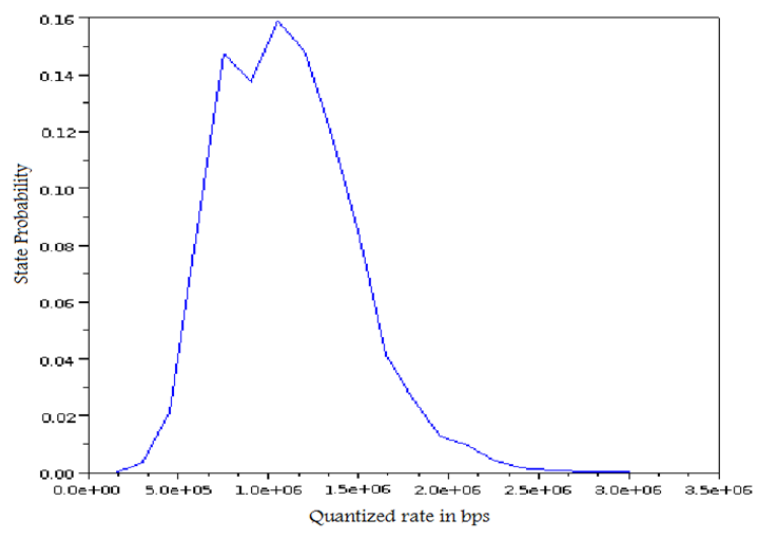

Figure 6. Obtained histogram to be mapped with the PDF function

Hence, we have the measurements that should be mapped to the states probability functions and using the state duration, the classification according to $\lambda$ and $\mu$ is performed. Then we can find the appropriate CAC. Actually, the measurement process should continue to update the system in case of strong variability even after the convergence. The states probabilities determination will then be time dependent as well as the CAC function.

As explained above, we used different repartitions of traffic flow characteristics, like talk shows, movies, news, soccer matches, etc. The obtained model flow characteristics for each category with the corresponding parameters are given in the table 1 given below.

\begin{tabular}{|c|c|c|}
\hline \multirow{2}{*}{ Model M1 } & $\lambda=0.8$ & Mean rate $=1.0$ \\
& $\mu=2.7$ & Mbps \\
\hline \multirow{2}{*}{ Model M2 } & $\lambda=0.6$ & Mean rate $=0.7$ \\
& $\mu=2.1$ & Mbps \\
\hline \multirow{2}{*}{ Model M3 } & $\lambda=0.5$ & Mean rate $=0.56$ \\
& $\mu=1.7$ & Mbps \\
\hline
\end{tabular}

Table 1. Flow characteristics measured for used traffic

Note that, for example, the soccer video game given should be mapped to the M1 model.

\section{B. CAC Performances}

The simulation using NS-2 simulator with the WiMAX module [11] is realised to assess the CAC performances. We changed the traffic flow amount, the traffic repartition according to the video characteristics and also the considered bandwidth. The goal of this simulation study is to show the performance obtained after adapting the CAC. We show that we can in some cases accept more flows in the system without QoS degradation and also, thanks to the CAC limitation and adaptation, how the QoS could be preserved.

We used different combinations of traffic flows. Those flows are those provided in [10] and we are able to change the distribution of traffics belonging to the different categories defined.

Once the flow characteristics and measurements obtained (after the convergence of the measurements), we can solve the Markov model, and hence obtain the CAC function output. Moreover, note that we also continuously perform the measurements to update the CAC even if no incoming flow is admitted, this to take into account the possible changes in the same video flow and hence make the corresponding changes if needed.

For the rtPS parameters, we set to 5 the number of 802.16 MAC frames per bandwidth request (denoted as respectively the $p$ and $f_{d}$ parameters in section III), with a MAC frame duration of $20 \mathrm{~ms}$. This leads us to set the duration of the quantization rate to scale time of $100 \mathrm{~ms}$. Moreover, to simplify the simulation problem, we focus only on the rtPS service with $10 \mathrm{Mbps}$ allocated bandwidth in the MAC frame.

Then, we choose 20 videos flows and with the characteristics $\eta_{1}, \eta_{2}, \eta_{3}$ of the following ratio values (from 1 to 5 ): $\{10 \%, 30 \%$, $60 \%\},\{20 \%, 30 \%, 50 \%\},\{30 \%, 30 \%, 40 \%\},\{30 \%, 50 \%$, $20 \%\},\{20 \%, 60 \%, 20 \%\}$ we compare the adaptive CAC to the case the where the CAC take into account only the mean value, with no distinction of the different video classes. The round values of $\eta_{1}, \eta_{2}, \quad \eta_{3}$ were obtained by adding/removing appropriate traffic loads. 


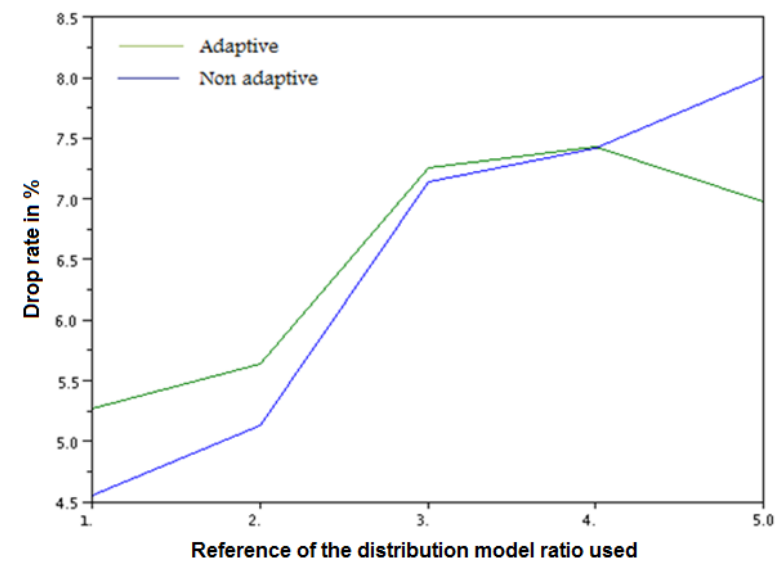

Figure 7. Comparative drop rate according to flows distribution

As depicted in Figure 7, we are able to maintain the drop rates very close to each other. This will allow us to admit more sources in the system as explained in the following part.

The CAC should limit the number of admitted sources to maintain the QoS above a threshold. Figure 8 gives the maximum number of admitted sources for a given threshold equal to $5 \%$ of losses. Note that the admission calculations were realised after having first computing the flows repartition.

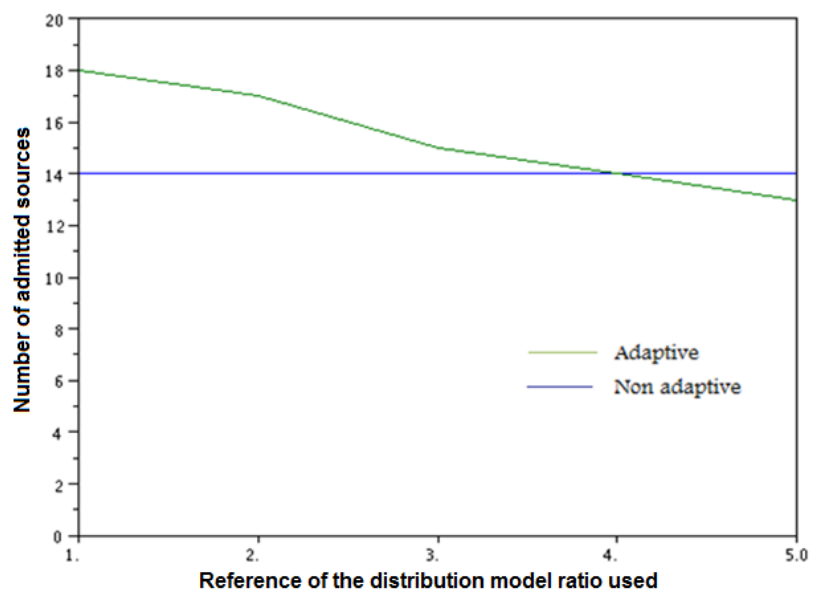

Figure 8. Number of Admissions $\mathrm{N}$ for adaptive and non adaptive CAC

As we can see, we were able to admit more sources thanks to the adaptation that was able to detect the possibility to admit more video flows without QoS degradation. However, we also detected that for the last simulation period, we were obliged to reduce the $\mathrm{CAC}$ in order to limit respect the limitation (drop rate).

\section{CONCLUSION AND FUTURE WORK}

In this paper we proposed an adaptive CAC based on measurements that classify incoming video flows according to the appropriate model they match. This classification gives categories of traffic of similar statistical behaviour and can be done in addition to model parameters refinement. Operator can enhance both the QoS (in term of drop for example) and the Grade of Service (GoS) in term of flow admission.

As a perspective to this work, we propose to take into account the SNR for CAC in WiMAX rtPS by adapting the bandwidth occupation in term of rate/time fairness. Moreover, we are also working on how to predict the bandwidth requests and then make a reservation in advance for priority video frames.

\section{REFERENCES}

[1] IEEE Std 802.16e, "IEEE Standard for Local and Metropolitan Area Networks Part 16: Air Interface for Fixed Broadband Wireless Access Systems", February 2006.

[2] An Efficient Call Admission Control for IEEE 802.16 Networks, MTechTechnical report, IIT, Bombay, 2006.

[3] A. Bianco, C. F. Chiasserini, E. Leonardi, "Bandwidth Allocation for Video Streaming in WiMax Networks", IEEE VTC 2009, Barcelona, April 2009.

[4] A. Belghith, L. Nuaymi, "Comparison of WiMAX scheduling algorithmsand proposals for the rtPS QoS class", European Wireless Conference, 22-25 June, 2008.

[5] Al Masri et al, "Proposal of a Novel Bandwidth Management Framework for IEEE 802.16 Based on Aggregation", NTMS, May 2009.

[6] C. H. Liew, A. M. Kondoz, M. Barbera, G. Schembra, "An Analytical Model for MPEG4 Transmission in Wireless Networks", EWCOM-ACoRN, September 2006.

[7] L. Lanfranchi and B. Bing, "MPEG-4 Bandwidth Prediction for Broadband Cable Networks", IEEE Transactions on Broadcasting, Fall 2008.

[8] H. Koumaraset al, "A Markov Modified Model of H.264 VBR Video Traffic", IST Mobile Summit 2006.

[9] C. Ke, C. Shieh, W. Hwang, A. Ziviani, "An Evaluation Framework for More Realistic Simulations of MPEG Video Transmission", Journal of Information Science and Engineering, 2008.

[10] http://www.tkn.tu-berlin.de/research/trace/trace.html

[11] J. Freitag, N.L.S da Fonseca, "Uplink Scheduling with Quality of Service in IEEE 802.16 Networks", Global Telecommunications Conference, december 2007. 